\title{
Oscillation criteria for a class of fractional delay differential equations
}

\author{
Pengxian Zhu ${ }^{1,2}$ and Qiaomin Xiang ${ }^{1,2^{*}}$
}

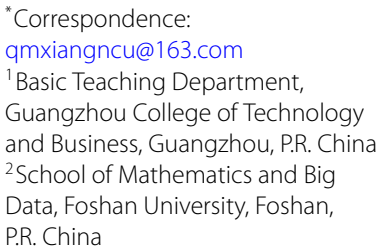

\begin{abstract}
This paper is devoted to the oscillatory problem in the fractional-order delay differential equations. First, we prove the convergence of the Laplace transform of a fractional operator by the generalized Gronwall inequality with singularity and fractional calculus technique. Then we show that it exhibits oscillation dynamics if the corresponding characteristic equation has no real roots. We further provide other direct and effective criteria depending on the system parameters and fractional exponent. Finally, we carry out some numerical simulations to illustrate our results.
\end{abstract}

MSC: 39A21;34A08

Keywords: Oscillation; Fractional differential equation; Delay

\section{Introduction}

Fractional calculus has drawn much attention in various fields of science and engineering over the past few decades [1-3]. Fractional derivatives provide an excellent instrument for the description of memory and hereditary properties of various materials and processes. This main advantage makes them useful to model some neglected effects with classical integer-order models. The list of applications of fractional calculus has been growing, including viscoelastic materials and rheology, electrical engineering, electrochemistry, biology, biophysics and bioengineering, signal and image processing, mechanics, mechatronics, physics, and control theory $[4,5]$.

On the other hand, delay differential equations are adopted to represent systems with time delay. Such effects arise in many processes, such as chemical processes (behaviors in chemical kinetics), technical processes (electric, pneumatic, and hydraulic networks), biosciences (heredity in population dynamics), economics (dynamics of business cycles), and other branches. The basic qualitative theory of these delay differential equations is well established, especially in the linear case (for general references, see [6-9]).

With the combination of both fractional derivative and time delay, the topic of fractionalorder delay differential equations (FDDEs) is enjoying growing interest among mathematicians and physicists. For instance, the general results on the existence of solutions of FDDEs were presented in [10]; the analytical stability bound for FDDEs was discussed in [11-13]; the finite time stability of robotic systems was studied in [14, 15], where time delay appears in $\mathrm{PD}^{\alpha}$ fractional control system; the necessary and sufficient conditions for asymptotic stability of $d$-dimensional linear FDDEs are obtained by using the inverse

(c) The Author(s) 2018. This article is distributed under the terms of the Creative Commons Attribution 4.0 International License (http://creativecommons.org/licenses/by/4.0/), which permits unrestricted use, distribution, and reproduction in any medium, provided you give appropriate credit to the original author(s) and the source, provide a link to the Creative Commons license, and indicate if changes were made. 
Laplace transform method in [16]; the stability and asymptotic properties of FDDEs were analyzed in [17].

To the best of our knowledge, however, very little is known regarding the oscillatory behavior of FDDEs (see [18-22]). For the first-order delay differential equation

$$
x^{\prime}(t)+p x(t-\tau)=0, \quad t>0,
$$

it is well known that the classical oscillation result characterized by parameters and fractional exponent is

$$
p \tau>\frac{1}{e}
$$

As is customary, a nontrivial solution of a differential equation is said to be oscillatory if eventually it is neither positive nor negative. Otherwise, the solution is called nonoscillatory. If all solutions of an equation are oscillatory, then this equation is said to be oscillatory.

The paper is organized as follows. In Sect. 2, we recall some concepts and facts, which are broadly used for deriving the main results of this paper. In Sect. 3, we present oscillation criteria for FDDEs of the form

$$
{ }^{\mathrm{C}} D_{t}^{\alpha} x(t)+p x(t-\tau)=0, \quad t>0,
$$

where ${ }^{\mathrm{C}} D_{t}^{\alpha}$ is the Caputo fractional derivative of order $0<\alpha<1$, and $p, \tau \in \mathbb{R}^{+}$. The standard initial condition associated with (1.1) is

$$
x(t)=\varphi(t), \quad t \in[-\tau, 0],
$$

where $\varphi(t) \in C([-\tau, 0], \mathbb{R})$. Precisely, we establish sufficient conditions for the oscillation via the parameter triple $(\alpha, p, \tau)$ in Theorem 3.2. In Sect. 4 , we further obtain sufficient conditions of the oscillation for FDDEs with positive and negative coefficients in Theorem 4.2. We provide two illustrative examples to demonstrate the applicability of the theoretical results in Sect. 5 .

\section{Preliminaries}

Let us first recall the necessary definitions of the fractional calculus.

Definition 2.1 The Riemann-Liouville fractional integral operator of order $\alpha$ of a function $f$ is defined as

$$
I^{\alpha} f(t)=\frac{1}{\Gamma(\alpha)} \int_{0}^{t}(t-s)^{\alpha-1} f(s) \mathrm{d} s, \quad t \geq 0,
$$

provided that the right side is pointwise defined on $[0, \infty)$, where $\Gamma(\cdot)$ is the gamma function. 
Definition 2.2 The Riemann-Liouville fractional derivative of order $\alpha$ of a function $f$ is defined as

$$
{ }^{\mathrm{RL}} D_{t}^{\alpha} f(t)=\frac{1}{\Gamma(1-\alpha)} \frac{\mathrm{d}}{\mathrm{d} t} \int_{0}^{t}(t-s)^{-\alpha} f(s) \mathrm{d} s, \quad t \geq 0 .
$$

Definition 2.3 The Caputo derivative of order $\alpha$ for a function $f$ is defined as

$$
{ }^{\mathrm{C}} D_{t}^{\alpha} f(t)=\frac{1}{\Gamma(1-\alpha)} \int_{0}^{t}(t-s)^{-\alpha} \frac{\mathrm{d}}{\mathrm{d} s} f(s) \mathrm{d} s={ }^{\mathrm{RL}} D_{t}^{\alpha}(f(t)-f(0)), \quad t \geq 0 .
$$

We further recall some facts about the Laplace transform, which play a significant role in the proof of the sufficient conditions for the oscillation of Eq. (1.1). Let $\mathcal{L}[\cdot]$ denote the Laplace transform. If $X(s)$ is the Laplace transform of $x(t)$,

$$
X(s)=\mathcal{L}[x(t)](s)=\int_{0}^{\infty} e^{-s t} x(t) \mathrm{d} t
$$

then the abscissa of convergence of $X(s)$ is defined as

$$
\sigma_{0}=\inf \{\sigma \in \mathbb{R}: X(\alpha) \text { exists }\}
$$

Therefore, $X(s)$ exists for $\operatorname{Re}(s)>\sigma_{0}$. The Laplace transform of the Caputo fractional derivative is given by [2]

$$
\mathcal{L}\left[{ }^{\mathrm{C}} D_{t}^{\alpha} x(t)\right](s)=s^{\alpha} X(s)-s^{\alpha-1} x(0) .
$$

Lemma 2.4 If $x(t)$ is the solution of Eq. (1.1), then $X(s)$ exists.

Proof We can rewrite Eq. (1.1) as the equivalent integral equation

$$
x(t)=\varphi(0)-\frac{p}{\Gamma(\alpha)} \int_{0}^{t}(t-s)^{\alpha-1} x(s-\tau) \mathrm{d} s, \quad t \geq 0,
$$

and $x(t)=\varphi(t)$ for $t \in[-\tau, 0]$.

If $t \in[-\tau, 0]$, then

$$
|x(t)| \leq\left(\frac{p t^{\alpha}}{\Gamma(\alpha+1)}\right)\|\varphi\|=: r(t)
$$

where $\|\varphi\|=\sup _{t \in[-\tau, 0]}|\varphi(t)|$.

If $t>\tau$, then we have

$$
|x(t)| \leq r(t)+\frac{p}{\Gamma(\alpha)} \int_{0}^{t}(t-s)^{\alpha-1}|x(s)| \mathrm{d} s .
$$

By the generalized Gronwall inequality with singularity [23] it follows that

$$
|x(t)| \leq r(t) E_{\alpha}\left(p t^{\alpha}\right)
$$

where $E_{\alpha}(\cdot)$ is the Mittag-Leffler function defined by $E_{\alpha}(z)=\sum_{k=0}^{\infty} \frac{z^{k}}{\Gamma(k \alpha+1)}$. 
Combining (2.3) with (2.2), we can see that $|x(t)| \leq r(t) E_{\alpha}\left(p t^{\alpha}\right)$ for $t \geq 0$. It is easy to show that $X(s)$ exists for $\operatorname{Re}(s)>p^{\frac{1}{\alpha}}$. The proof is complete.

\section{Oscillation criteria for FDDEs}

Now we are in position to give our main results.

Theorem 3.1 Assume that $p, \tau \in \mathbb{R}^{+}$, and let $\alpha \in(0,1)$ be the ratio of two odd integers. If the equation

$$
F(\lambda)=\lambda^{\alpha}+p e^{-\lambda \tau}=0
$$

has no real roots, then every solution of Eqs. (1.1)-(1.2) oscillates.

Proof Suppose that $x(t)$ is a nonoscillatory solution of Eq. (1.1). Without loss of generality, we assume that $x(t)$ is an eventually positive solution of Eq. (1.1), that is, there exists a positive constant $T$ such that $x(t)>0$ for $t>T$. As Eq. (1.1) is autonomous, we may assume that $x(t)>0$ for $t \geq-\tau$. By Lemma 2.4 we can take the Laplace transform of both sides of Eq. (1.1), which gives for $\operatorname{Re}(s)>\sigma_{0}$ :

$$
s^{\alpha} X(s)-s^{\alpha-1} x(0)+p e^{-s \tau} X(s)+p e^{-s \tau} \int_{-\tau}^{0} e^{-s t} x(t) \mathrm{d} t=0
$$

that is,

$$
\left(s^{\alpha}+p e^{-s \tau}\right) X(s)=s^{\alpha-1} x(0)-p e^{-s \tau} \int_{-\tau}^{0} e^{-s t} x(t) \mathrm{d} t=0 .
$$

Let

$$
\Phi(s)=s^{\alpha-1} x(0)-p e^{-s \tau} \int_{-\tau}^{0} e^{-s t} x(t) \mathrm{d} t
$$

Then we have

$$
F(s) X(s)=\Phi(s), \quad \operatorname{Re}(s)>\sigma_{0} .
$$

It is obvious that $F(s)$ and $\Phi(s)$ are entire functions. Since Eq. (3.1) has no real roots and $F(0)=p>0$, we conclude that $F(s)>0$ for $s \in \mathbb{R}$. Furthermore, $\sigma_{0}=-\infty$ (see [6, Theorem 2.1.1]). Thus, we can deduce from (3.3) that

$$
X(s)=\frac{\Phi(s)}{F(s)} \quad \text { for all } s \in \mathbb{R} .
$$

By taking $s \rightarrow-\infty$ we can see that (3.4) leads to a contradiction because $X(s)$ and $F(s)$ are always positive whereas $\Phi(s)$ becomes eventually negative by (3.2). Indeed, it follows from (2.1) and the fact $x(t)>0$ for $t>0$ that $X(s)$ is positive and $F(s)>0$ for all negative real $s$. By the positivity of $x(t)$ on $[-\tau, 0]$ and $\lim _{s \rightarrow-\infty} s^{\alpha-1} \rightarrow 0$ we have $\lim _{s \rightarrow-\infty} \Phi(s)=-\infty$. The proof is complete.

Having the above result, we further obtain sufficient conditions of oscillation for Eq. (1.1). 
Theorem 3.2 Assume that $p, \tau \in \mathbb{R}^{+}$, and let $\alpha \in(0,1)$ be the ratio of two odd integers. If

$$
\tau p^{\frac{1}{\alpha}}>\frac{1}{e}
$$

then every solution of Eq. (1.1) oscillates.

Proof Our goal is to prove that Eq. (3.1) has no real roots due to Theorem 3.1. Assume, for contradiction, that Eq. (3.1) has a negative root $\lambda$. In fact, if $\lambda \geq 0$, then $\lambda^{\alpha}+p e^{-\lambda \tau}>0$. Without loss of generality, let $\lambda_{1}=-\lambda>0$. Since $\alpha$ is the ratio of two odd integers, it follows from (3.1) that

$$
\lambda_{1}^{\alpha}=p e^{\lambda_{1} \tau} \geq p
$$

By (3.5) and the inequality $e^{x} \geq e x$ for $x \geq 0$ we can see that

$$
\lambda_{1}^{\alpha}=p e^{\lambda_{1} \tau} \geq \lambda_{1} p \tau e=\lambda_{1}^{\alpha} \lambda_{1}^{1-\alpha} p \tau e \geq \lambda_{1}^{\alpha} p^{\frac{1}{\alpha}} \tau e,
$$

which implies $\tau p^{\frac{1}{\alpha}} \leq \frac{1}{e}$, and this is a contradiction. The proof is complete.

Consider the linear nonautonomous FDDEs

$$
{ }^{\mathrm{C}} D_{t}^{\alpha} x(t)+P(t) x(t-\tau)=0, \quad t>0,
$$

with the initial condition (1.2), where $P(t) \in C\left((0, \infty), \mathbb{R}^{+}\right)$.

Corollary 3.3 Assume that $\tau \in \mathbb{R}^{+}$and that $\alpha \in(0,1)$ be the ratio of two odd integers. If

$$
\liminf _{t \rightarrow+\infty} P(t)=p>0
$$

and

$$
\tau p^{\frac{1}{\alpha}}>\frac{1}{e}
$$

then every solution of Eq. (3.6) oscillates.

Proof Assume, on the contrary, that Eq. (3.1) has an eventually positive solution $x(t)$, that is, there exists a sufficiently large positive constant $T$ such that $x(t)>0$ and $x(t-\tau)>0$ for $t>\tau$. It follows from (3.7) that

$$
\begin{aligned}
0 & ={ }^{C} D_{t}^{\alpha} x(t)+P(t) x(t-\tau) \geq{ }^{C} D_{t}^{\alpha} x(t)+\lim \inf _{t \rightarrow+\infty} P(t) x(t-\tau) \\
& ={ }^{C} D_{t}^{\alpha} x(t)+p x(t-\tau), \quad t>T .
\end{aligned}
$$

Then we can see that the eventually positive solution $x(t)$ satisfies the inequality

$$
{ }^{\mathrm{C}} D_{t}^{\alpha} x(t)+p x(t-\tau) \leq 0, \quad t>T .
$$


According to (3.8) and Theorem 3.2, it follows that Eq. (3.1) has no real roots. Therefore, similarly to the proof of Theorem 3.1, inequality (3.9) has no eventually positive solution, which implies that every solution of Eq. (3.6) oscillates.

\section{Oscillation criteria for FDDEs with two constant coefficients}

In this section, we consider FDDEs with positive and negative coefficients of the form

$$
{ }^{\mathrm{C}} D_{t}^{\alpha} x(t)+p x(t-\tau)+q x(t)=0, \quad t>0,
$$

where $p, \tau \in \mathbb{R}^{+}$and $q \in \mathbb{R}$.

Proposition 4.1 Assume that $q, p, \tau \in \mathbb{R}^{+}$and that $\alpha \in(0,1)$ is the ratio of two odd integers. If the equation

$$
\lambda^{\alpha}+p e^{-\lambda \tau}+q=0
$$

has no real roots, then every solution of Eq. (4.1) oscillates.

Proof The proof of this result is similar to that of Theorem 3.1, and thus we omit it.

Theorem 4.2 Assume that $q, p, \tau \in \mathbb{R}^{+}$and that $\alpha \in(0,1)$ is the ratio of two odd integers. If

$$
p \leq q \text { and } \frac{p \tau}{(q+p)^{\frac{\alpha-1}{\alpha}}+q \tau}>\frac{1}{e}
$$

then every solution of Eq. (4.1) oscillates.

Proof Assume that (4.3) holds and, for contradiction, assume that Eq. (4.2) has a negative root $\lambda_{1}$. Then, in view of (4.2), we have

$$
\lambda_{1}\left(\lambda_{1}^{\alpha-1}+q \int_{0}^{\tau} e^{\lambda_{1} s} \mathrm{~d} s\right)=\lambda_{1}^{\alpha}+q\left(e^{\lambda_{1} \tau}-1\right)=-p e^{-\lambda_{1} \tau}-q\left(2-e^{\lambda_{1} \tau}\right)
$$

and

$$
\lambda_{1}^{\alpha}=-q-p e^{-\lambda_{1} \tau}<-(q+p) .
$$

Since $\alpha=$ odd integer/odd integer, from (4.5) we have

$$
\lambda_{1}^{\alpha-1}<(q+p)^{\frac{\alpha-1}{\alpha}}
$$

Consequently, from (4.4) and (4.6) it follows that

$$
0<\lambda_{1}^{\alpha-1}+q \int_{0}^{\tau} e^{\lambda_{1} s} \mathrm{~d} s<\lambda_{1}^{\alpha-1}+q \tau<(q+p)^{\frac{\alpha-1}{\alpha}}+q \tau .
$$


Then, using (4.4) and (4.7), we obtain

$$
\begin{aligned}
0 & =\lambda_{1}\left(\lambda_{1}^{\alpha-1}+q \int_{0}^{\tau} e^{\lambda_{1} s} \mathrm{~d} s\right)+p e^{-\lambda_{1} \tau}+q\left(2-e^{\lambda_{1} \tau}\right) \\
& >\left[(q+p)^{\frac{\alpha-1}{\alpha}}+q \tau\right] \lambda_{1}+p e^{-\lambda_{1} \tau}+q\left(2-e^{\lambda_{1} \tau}\right),
\end{aligned}
$$

which implies

$$
\left[(q+p)^{\frac{\alpha-1}{\alpha}}+q \tau\right] \lambda_{1}+p e^{-\lambda_{1} \tau}<0 .
$$

Let

$$
G(\lambda)=\lambda+\frac{p}{(q+p)^{\frac{\alpha-1}{\alpha}}+q \tau} e^{-\lambda \tau}=0
$$

Since $G(0)=\frac{p}{(q+p)^{\frac{\alpha-1}{\alpha}}+q \tau}>0$ and $G\left(\lambda_{1}\right)<0, G(\lambda)$ has a real root in $\left(\lambda_{1}, 0\right)$ by the intermediate value theorem. Thus, according to the classical oscillation criteria of first-order delay differential equation (see e.g., [6]), it follows that

$$
\frac{p \tau}{(q+p)^{\frac{\alpha-1}{\alpha}}+q \tau} \leq \frac{1}{e}
$$

This leads to a contradiction.

Corollary 4.3 Assume that $q, p, \tau \in \mathbb{R}^{+}$and that $\alpha \in(0,1)$ is the ratio of two odd integers. If

$$
p>q \text { and } \frac{p \tau}{(q+p)^{\frac{\alpha-1}{\alpha}}+p \tau}>\frac{1}{e}
$$

then every solution of Eq. (4.1) oscillates.

Proof Assume that (4.9) holds and, for contradiction, assume that Eq. (4.2) has a negative root $\lambda_{1}$. Then, in view of (4.2), we have

$$
\lambda_{1}\left(\lambda_{1}^{\alpha-1}+q \int_{0}^{\tau} e^{\lambda_{1} s} \mathrm{~d} s\right)=\lambda_{1}^{\alpha}+p\left(e^{\lambda_{1} \tau}-1\right)=-p e^{-\lambda_{1} \tau}-q-p\left(1-e^{\lambda_{1} \tau}\right) .
$$

The remainder of the argument is analogous to that in the proof of Theorem 4.2, so it is omitted.

Corollary 4.4 Assume that $p, \tau \in \mathbb{R}^{+}, q \in \mathbb{R}^{-}$and that $\alpha \in(0,1)$ is the ratio of two odd integers. If

$$
p>-q, \quad(q+p)^{\frac{\alpha-1}{\alpha}}>-q \tau \quad \text { and } \quad \frac{(p+q) \tau}{(q+p)^{\frac{\alpha-1}{\alpha}}+q \tau}>\frac{1}{e}
$$

then every solution of Eq. (4.1) oscillates. 
Proof Assume that (4.10) holds and, for contradiction, assume that Eq. (4.2) has a negative $\operatorname{root} \lambda_{1}$. Then

$$
\lambda_{1}\left(\lambda_{1}^{\alpha-1}+q \int_{0}^{\tau} e^{-\lambda_{1} s} \mathrm{~d} s\right)=\lambda_{1}^{\alpha}+q\left(e^{-\lambda_{1} \tau}-1\right)=-(p+q) e^{-\lambda_{1} \tau} .
$$

Consequently, by (4.11) and (4.6) we obtain

$$
0<\lambda_{1}^{\alpha-1}+q \int_{0}^{\tau} e^{-\lambda_{1} s} \mathrm{~d} s<\lambda_{1}^{\alpha-1}+q \tau<(q+p)^{\frac{\alpha-1}{\alpha}}+q \tau .
$$

Therefore, it follows from (4.11) and (4.12) that

$$
\left[(q+p)^{\frac{\alpha-1}{\alpha}}+q \tau\right] \lambda_{1}+p e^{-\lambda_{1} \tau}<0
$$

The remainder of the argument is analogous to that in the proof of Theorem 4.2.

\section{Numerical simulation}

To illustrate the effectiveness and the flexibility of our theoretical analysis, we give two numerical examples.

\subsection{Numerical algorithm}

The numerical methods used for solving ordinary differential equations cannot be used directly to solve fractional differential equations because of the nonlocal nature of fractional differential equations. Diethelm et al. [24] proposed a numerical algorithm for solving fractional differential equations. This scheme is a generalization of the Adams-BashforthMoulton method, that is, the predictor-corrector approach. Recently, Bhalekar and Daftardar-Gejji [25] have extended this algorithm to solve FDDEs.

Consider the following FDDE:

$$
\left\{\begin{array}{l}
{ }^{C} D_{t}^{\alpha} x(t)+f(x(t), x(t-\tau))=0, \quad t>0, \\
x(t)=\varphi(t), \quad t \in[-\tau, 0] .
\end{array}\right.
$$

Assume that we are working on a uniform grid $\left\{t_{n}=n h: n=-k,-k+1, \ldots,-1,0,1, \ldots, N\right\}$, where $k$ and $N$ are integers such that $h=\frac{T}{N}$ and $h=\frac{\tau}{k}$. Let

$$
x_{h}\left(t_{j}\right)=\varphi\left(t_{j}\right), \quad j=-k,-k+1, \ldots,-1,0,
$$

and note that

$$
x_{h}\left(t_{j}-\tau\right)=x_{h}(j h-k h)=x_{h}\left(t_{j-k}\right), \quad j=0,1, \ldots, N .
$$

Suppose that we have already calculated approximations

$$
x_{h}\left(t_{j}\right) \approx x\left(t_{j}\right), \quad j=-k,-k+1, \ldots,-1,0,1, \ldots, n,
$$

and want to calculate $x_{h}\left(t_{n}+1\right)$ using

$$
x\left(t_{n+1}\right)=\varphi(0)-\frac{1}{\Gamma(\alpha)} \int_{0}^{t_{n+1}}\left(t_{n+1}-s\right)^{\alpha-1} f(x(s), x(s-\tau)) \mathrm{d} s .
$$


We use approximations $x_{h}\left(t_{n}\right)$ for $x\left(t_{n}\right)$ in (5.1). Furthermore, we evaluate the integral in (5.1) by the product trapezoidal quadrature formula. The corrector formula is thus

$$
\begin{aligned}
x_{h}\left(t_{n+1}\right)= & \varphi(0)-\frac{h^{\alpha}}{\Gamma(\alpha+2)} f\left(x_{h}^{p}\left(t_{n+1}\right), x_{h}\left(t_{n+1}-\tau\right)\right) \\
& -\frac{h^{\alpha}}{\Gamma(\alpha+2)} \sum_{j=0}^{n} a_{j, n+1} f\left(x_{h}\left(t_{j}\right), x_{h}\left(t_{j}-\tau\right)\right) \\
= & \varphi(0)-\frac{h^{\alpha}}{\Gamma(\alpha+2)} f\left(x_{h}^{p}\left(t_{n+1}\right), x\left(t_{n+1-k}\right)\right) \\
& -\frac{h^{\alpha}}{\Gamma(\alpha+2)} \sum_{j=0}^{n} a_{j, n+1} f\left(x_{h}\left(t_{j}\right), x_{h}\left(t_{j-k}\right)\right),
\end{aligned}
$$

where

$$
a_{j, n+1}= \begin{cases}n^{\alpha}+1-(n-\alpha)(n+1)^{\alpha}, & j=0 \\ (n-j+2)^{\alpha+1}+(n-j)^{\alpha+1}-2(n-j+1)^{\alpha+1}, & 1 \leq j \leq n \\ 1, & j=n+1\end{cases}
$$

The preliminary approximation $x_{h}^{p}\left(t_{n+1}\right)$ is called the predictor and is given by

$$
\begin{aligned}
x_{h}^{p}\left(t_{n+1}\right) & =\varphi(0)-\frac{1}{\Gamma(\alpha)} \sum_{j=0}^{n} b_{j, n+1} f\left(x_{h}\left(t_{j}\right), x_{h}\left(t_{j}-\tau\right)\right) \\
& =\varphi(0)-\frac{1}{\Gamma(\alpha)} \sum_{j=0}^{n} b_{j, n+1} f\left(x_{h}\left(t_{j}\right), x_{h}\left(t_{j-k}\right)\right),
\end{aligned}
$$

where

$$
b_{j, n+1}=\frac{h^{\alpha}}{\alpha}\left((n+1-j)^{\alpha}-(n-j)^{\alpha}\right) .
$$

\subsection{Numerical results}

Example 5.1 Let the system parameters $(\alpha, p, \tau)=(0.6,1.15,2)$ for Eq. $(1.1)$. It is clear that

$$
\tau p^{\frac{1}{\alpha}} \approx 2.5246>\frac{1}{e}
$$

Thus all the solutions of Eq. (1.1) are oscillatory due to Theorems 3.1 and 3.2. However, if we take $(\alpha, p, \tau)=(0.6,0.2,2)$, then

$$
\tau p^{\frac{1}{\alpha}} \approx 0.1368<\frac{1}{e} .
$$

Taking the initial condition $\varphi(t)=0.5$ for $t \in[-2,0]$, the step-length $h=0.01$, and the iterated time $N=10,000$, we can observe that the solution of Eq. (1.1) is oscillatory when $(\alpha, p, \tau)=(0.6,1.15,2)$ and non-oscillatory when $(\alpha, p, \tau)=(0.6,0.2,2)$, as shown in Fig. 1. 


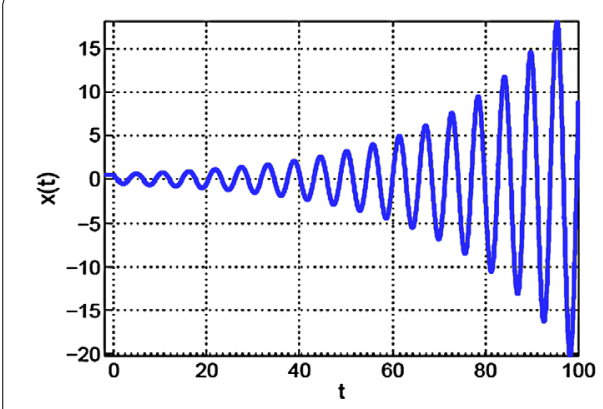

(a) Oscillatory solution of Eq. (1.1) with $(\alpha, p, \tau)=(0.6,1.15,2)$

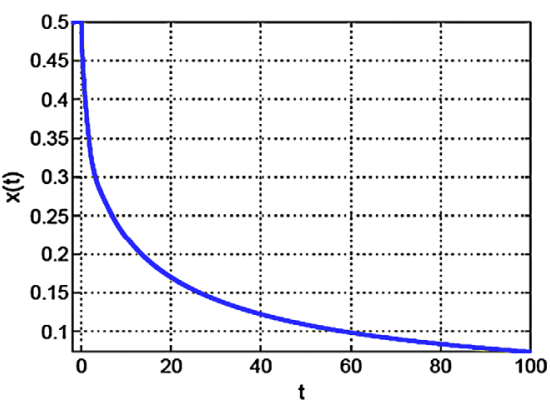

(b) Non-oscillatory solution of Eq. (1.1) with $(\alpha, p, \tau)=(0.6,0.2,2)$

Figure 1 Oscillatory behavior of Eq. (1.1)

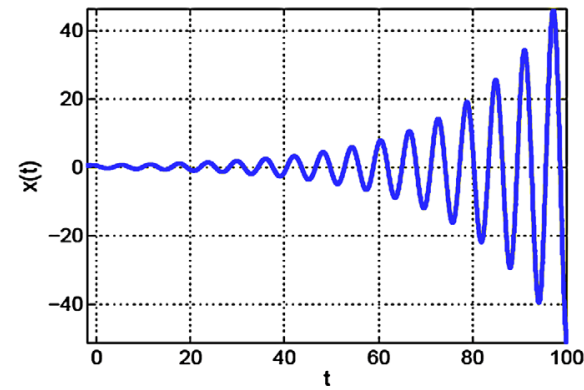

(a) Oscillatory solution of Eq. (4.1) with $(\alpha, p, q, \tau)=(0.6,1,0.2,2)$

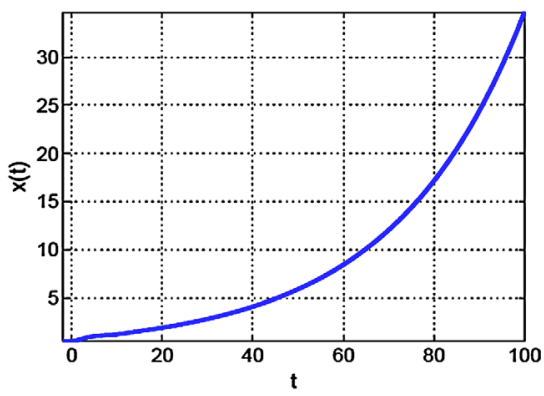

(b) Non-oscillatory solution of Eq. (4.1) with $(\alpha, p, q, \tau)=(0.6,0.5,0.6,2)$

Figure 2 Oscillatory behavior of Eq. (4.1)

Example 5.2 Let the system parameters $(\alpha, p, q, \tau)=(0.6,1,0.2,2)$ for Eq. (4.1). By calculations we have

$$
p>q, \quad(q-p)^{\frac{\alpha-1}{\alpha}}>q \tau \text { and } \frac{(p-q) \tau}{(q-p)^{\frac{\alpha-1}{\alpha}}-q \tau} \approx 2.1042>\frac{1}{e} .
$$

Therefore, by Theorems 4.1 and 4.2 all the solutions of Eq. (4.1) are oscillatory. Moreover, if $(\alpha, p, q, \tau)=(0.6,0.5,0.6,2)$, then the first condition in Theorem 4.2 is not satisfied. We continue to use the setting as in the previous example and also observe that the solution of Eq. (4.1) is oscillatory when $(\alpha, p, q, \tau)=(0.6,1,0.2,2)$ and non-oscillatory when $(\alpha, p, q, \tau)=(0.6,0.5,0.6,2)$, as shown in Fig. 2.

\section{Conclusion}

In this paper, we studied the oscillatory behavior of solutions of FDDEs. We have proved that FDDEs exhibit oscillation dynamics if the corresponding characteristic equation has no negative real roots. We further give some sufficient conditions for the oscillation of FDDEs based on parameters and fractional exponent, which are very convenient for using in practice. Nevertheless, necessary conditions for the oscillation of FDDEs and oscillatory criteria for nonlinear FDDEs remain open. They will be the subjects of our further investigation. 
Acknowledgements

The authors would like to thank reviewers for their insightful and valuable comments.

\section{Funding}

Not applicable.

\section{Competing interests}

The authors declare that they have no competing interests.

\section{Authors' contributions}

The authors have achieved equal contributions. Both authors read and approved the manuscript.

\section{Publisher's Note}

Springer Nature remains neutral with regard to jurisdictional claims in published maps and institutional affiliations.

Received: 2 July 2018 Accepted: 24 September 2018 Published online: 31 October 2018

\section{References}

1. Oldham, K.B., Spanier, J.: The Fractional Calculus. Academic Press, New York (1974)

2. Podlubny, I.: Fractional Differential Equations. Academic Press, San Diego (1999)

3. Hilfer, R:: Applications of Fractional Calculus in Physics. World Scientific, River Edge (2001)

4. Monje, C.A., Chen, Y.Q., Vinagre, B.M., Xue, D., Feliu, V.: Fractional-Order Systems and Controls: Fundamentals and Applications. Springer, London (2010)

5. Ortigueira, M.D.: Fractional Calculus for Scientists and Engineers. Springer, Berlin (2011)

6. Györi, I., Ladas, G.: Oscillation Theory of Delay Differential Equations with Applications. Oxford University Press, Oxford (1991)

7. Hale, J.K. Verduyn Lunel, S.M.: Introduction to Functional Differential Equations. Springer, New York (1993)

8. Kuang, Y.: Delay Differential Equations with Applications in Population Dynamics. Academic Press, Boston (1993)

9. Kolmanovskii, V., Myshkis, A.: Introduction to the Theory and Applications of Functional Differential Equations. Kluwer Academic, Dordrecht (1999)

10. Lakshmikantham, V.: Theory of fractional functional differential equations. Nonlinear Anal. 69, 3337-3343 (2008)

11. Chen, Y., Moore, K.L.: Analytical stability bound for a class of delayed fractional order dynamic systems. Nonlinear Dyn. 29, 191-200 (2002)

12. Hwang, C., Cheng, Y.C.: A numerical algorithm for stability testing of fractional delay systems. Automatica $\mathbf{4 2}$ 825-831 (2006)

13. Deng, W., Li, C., Lü, J.: Stability analysis of linear fractional differential system with multiple time delays. Nonlinear Dyn. 48, 409-416 (2007)

14. Lazarević, M.P.: Finite time stability analysis of $\mathrm{PD}^{\alpha}$ fractional control of robotic time-delay systems. Mech. Res. Commun. 33, 269-279 (2006)

15. Lazarević, M.P., Spasić, A.M.: Finite-time stability analysis of fractional order time delay systems: Gronwall's approach. Math. Comput. Model. 49, 475-481 (2009)

16. Krol, K:: Asymptotic properties of fractional delay differential equations. Appl. Math. Comput. 218, 1515-1532 (2011)

17. Čermák, J., Horní̌ek, J., Kisela, T.: Stability regions for fractional differential systems with a time delay. Commun. Nonlinear Sci. Numer. Simul. 31, 108-123 (2016)

18. Chen, D.X:: Oscillation criteria of fractional differential equations. Adv. Differ. Equ. 2012, Article ID 33 (2012)

19. Bolat, Y.: On the oscillation of fractional-order delay differential equations with constant coefficients. Commun. Nonlinear Sci. Numer. Simul. 19, 3988-3993 (2014)

20. Wang, Y., Han, Z., Sun, S.: Comment on "On the oscillation of fractional-order delay differential equations with constant coefficients" [Commun Nonlinear Sci 19(11) (2014) 3988-3993]. Commun. Nonlinear Sci. Numer. Simul. 26(1-3), 195-200 (2015)

21. Zhou, Y., Ahmad, B., Chen, F., Alsaedi, A.: Oscillation for fractional partial differential equations. Bull. Malays. Math. Soc. (2017). https://doi.org/10.1007/s40840-017-0495-7

22. Zhou, Y., Alsaedi, A., Ahmad, B.: Oscillation for fractional neutral functional differential systems. J. Comput. Anal. Appl. 25(5), 965-974 (2018)

23. Ye, H.P., Gao, J.M., Ding, Y.S.: A generalized Gronwall inequality and its application to a fractional differential equation. J. Math. Anal. Appl. 328, 1075-1081 (2007)

24. Diethelm, K., Ford, N., Freed, A.: A predictor-corrector approach for the numerical solution of fractional differential equations. Nonlinear Dyn. 29, 3-22 (2002)

25. Bhalekar, S., Daftardar-Gejji, V: A predictor-corrector scheme for solving nonlinear delay differential equations of fractional order. J. Fract. Calc. Appl. 1, Article ID 5 (2011) 